\title{
UCRL-CONF-207026
}

LAW RENCE LIVERMORE N A T IO N A L LABORATORY

\section{Optimization and Improvement of FOA Corner Cube Algorithm}

W. A. McClay, A. A. S. Awwal, S. C. Burkhart, J. V. Candy

October 4, 2004

SPIE, Annual Meeting 2004

Denver, CO, United States August 2, 2005 through August 5, 2005 
This document was prepared as an account of work sponsored by an agency of the United States Government. Neither the United States Government nor the University of California nor any of their employees, makes any warranty, express or implied, or assumes any legal liability or responsibility for the accuracy, completeness, or usefulness of any information, apparatus, product, or process disclosed, or represents that its use would not infringe privately owned rights. Reference herein to any specific commercial product, process, or service by trade name, trademark, manufacturer, or otherwise, does not necessarily constitute or imply its endorsement, recommendation, or favoring by the United States Government or the University of California. The views and opinions of authors expressed herein do not necessarily state or reflect those of the United States Government or the University of California, and shall not be used for advertising or product endorsement purposes. 


\title{
Optimization and Improvement of FOA Corner Cube Algorithm
}

\author{
Wilbert A. McClay, Abdul Awwal, Scott Burkhart, and James V. Candy \\ Lawrence Livermore National Laboratory, Livermore, CA. 94551 \\ E-mail: mcclay2@1lnl.gov
}

\begin{abstract}
Alignment of laser beams based on video images is a crucial task necessary to automate operation of the 192 beams at the National Ignition Facility (NIF). The final optics assembly (FOA) is the optical element that aligns the beam into the target chamber. This work presents an algorithm for determining the position of a corner cube alignment image in the final optics assembly. The improved algorithm was compared to the existing FOA algorithm on 900 noise-simulated images. While the existing FOA algorithm based on correlation with a synthetic template has a radial standard deviation of 1 pixel, the new algorithm based on classical matched filtering (CMF) and polynomial fit to the correlation peak improves the radial standard deviation performance to less than 0.3 pixels. In the new algorithm the templates are designed from real data stored during a year of actual operation.
\end{abstract}

\section{INTRODUCTION}

The National Ignition Facility, currently under construction at the Lawrence Livermore National Laboratory, is a stadium-sized facility containing a 192-beam, 1.8-megajoule, 500-terawatt, ultraviolet laser system for the study of inertial confinement fusion and the physics of matter at extreme energy densities and pressures [1]. The FOA is the last element of the NIF main laser system and the first of the target area systems. The main function of the FOA is to convert the infrared laser light to ultraviolet and focus it on to the target. An FOA Corner Cube can be described as a retro reflector that positions the laser beam at the target as the laser travels through a vacuum window. The determination of the optimal centroid position of the FOA Corner Cube images has been a long-standing challenge for the automatic alignment of the Final Optics Assembly at NIF.

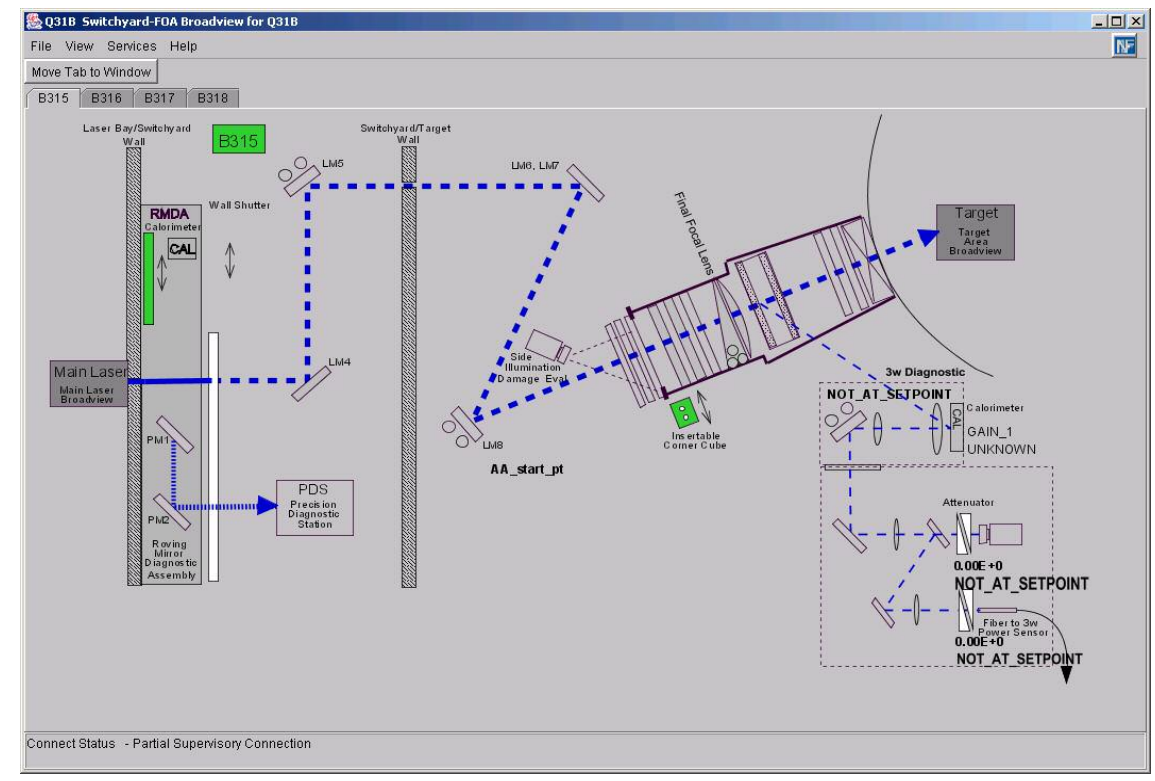

Figure 1. Final optics assembly graphical control interface 
The initial step in performing main laser beam alignment is to establish a reference position at beam center. An image of the reference is captured by a CCD video camera as shown in Figure 2a. Next a sequence of measurements is made using the alignment laser source. The laser fires creating a test beam that propagates through a sequence of mirrors through the spatial filter to the FOA. Here two corner cube ${ }^{1}$ reflectors reflect the beam back to the CCD camera where the "FOA alignment image" is produced. The image in Figure $2 \mathrm{~b}$ consists (ideally) of two circles with vertically spaced, diagonal lines; the position estimate is taken as the bisector of a line segment through the centers. The function of the FOA algorithm is to find the centroids of the two FOA corner cube images within 0.5 pixels.

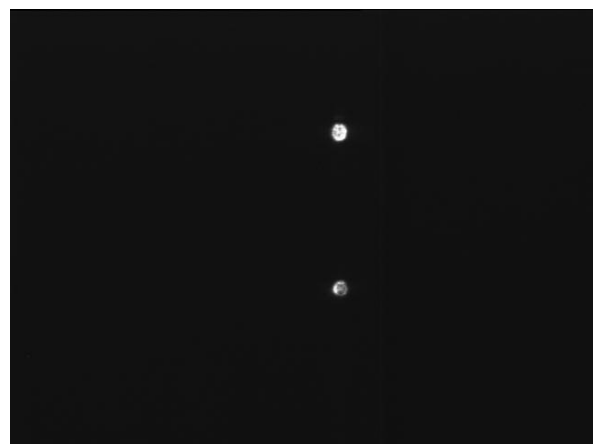

Figure 2a. Reference image

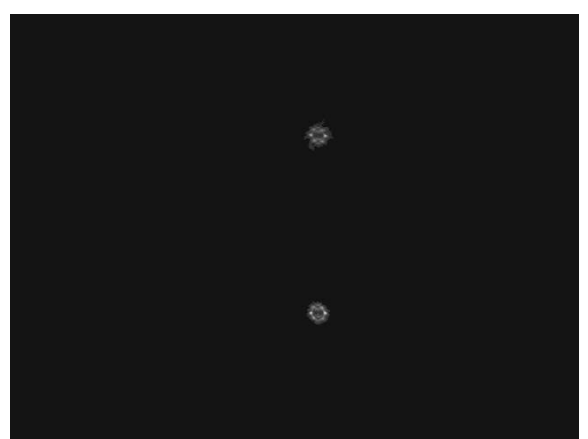

Figure 2b. FOA alignment image

\section{BACKGROUND}

A classical 2-D matched filter is a 2-D crosscorrelation where a template match is known as a 2-D autocorrelation. A template is defined as the reference image where a given digital image is noted as $f(x, y)$ and the size of the image is defined as $M \times N$. The goal is to find a region within the digital image similar to $w(x, y)$ with a size of $\mathrm{p} \times \mathrm{q}$, where $\mathrm{p}$ $<\mathrm{M}$ and $\mathrm{q}<\mathrm{N}$. The 2-D cross correlation is defined between the template or reference image $R(x, y)$ and the noise image, $Z(x, y)$. The equation defining the relationship between the template and reference image is $Z(x, y)=R(x, y)+N(x, y)$, where $N(x, y)$ is additive noise. Thus, the crosscorrelation between $Z(x, y)$ and $R(x, y)$ is $C_{Z R}(x-\Delta x, y-\Delta y)$ is defined in Eq. (1).

$$
E\left\{Z(x, y) R(x-\Delta x, y-\Delta y\}=C_{Z R}(x-\Delta x, y-\Delta y)\right.
$$

The 2-D autocorrelation is at a maximum at $C_{R R}(0,0)$, and the matched filter solution gives the location as:

$$
(\hat{x}, \hat{y})=(\Delta x, \Delta y)
$$

\footnotetext{
${ }^{1}$ A corner cube reflector is a cube that is cut to have three mutually perpendicular faces with the property that it is retrodirective, that is, it reflects all incoming rays back along their original directions.
} 


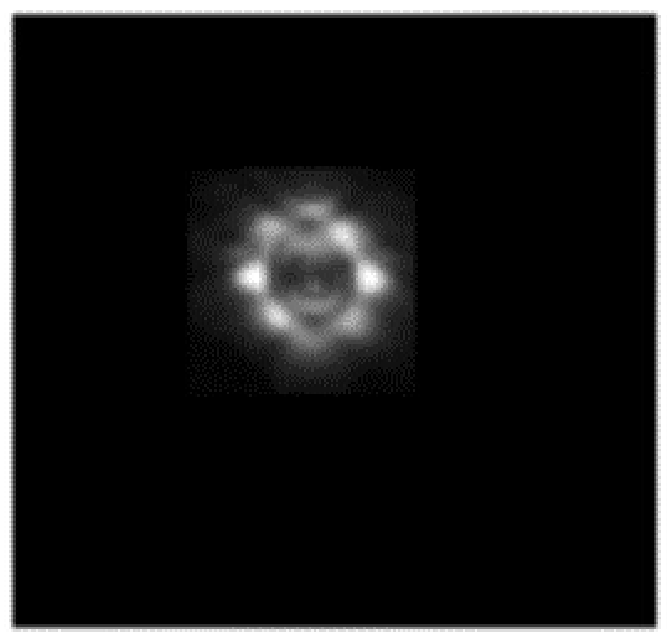

Figure 3. Template image formed by averaging $100+$ real images

The correlation is performed in the Fourier domain by multiplying the complex conjugate of the Fourier transformed reference with the transform of the input image. The peak is detected by interpolating a second order polynomial. The displacement of the template from its original location is used to find the location of the object.

\section{TEMPLATE FORMULATION}

The robustness of the proposed algorithm using the classical match filter depends heavily on the choice of an optimum template. For each beamline with an initial reference template, the optimal template is designed by averaging the data over an annual period where the criterion for an optimal template is decided by images that lie within the boundaries of the two-sigma ellipsoid. The graphs for two typical beams, the two-sigma scatter plots, and the two-sigma ellipse confidence intervals are shown in Figures 4 and 5. It should be noted that the images that lie inside the ellipsoid are registered (with respect to each other) to a common center location and averaged. 


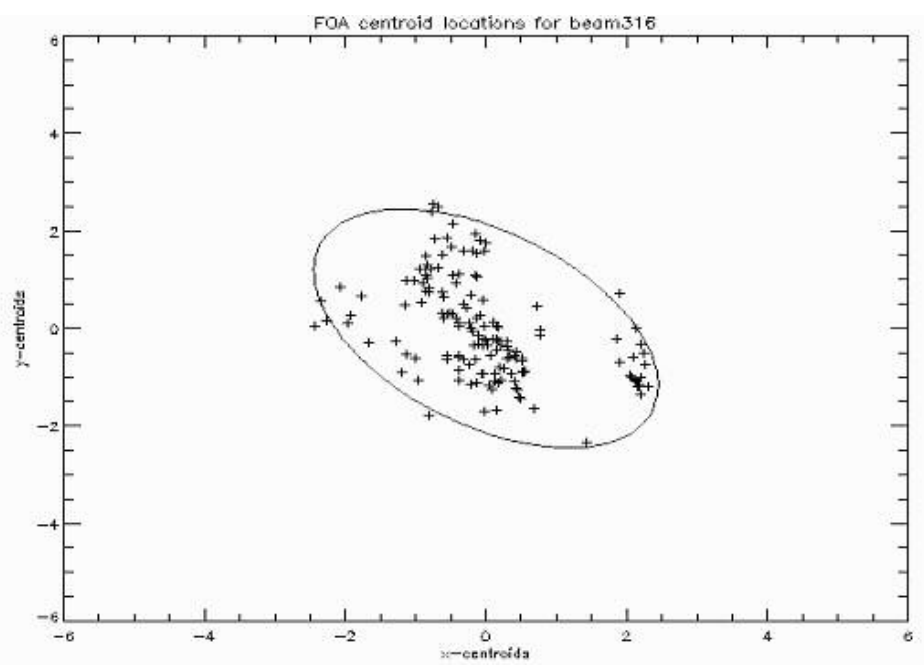

Figure 4. Two-sigma confidence ellipse for beam 316

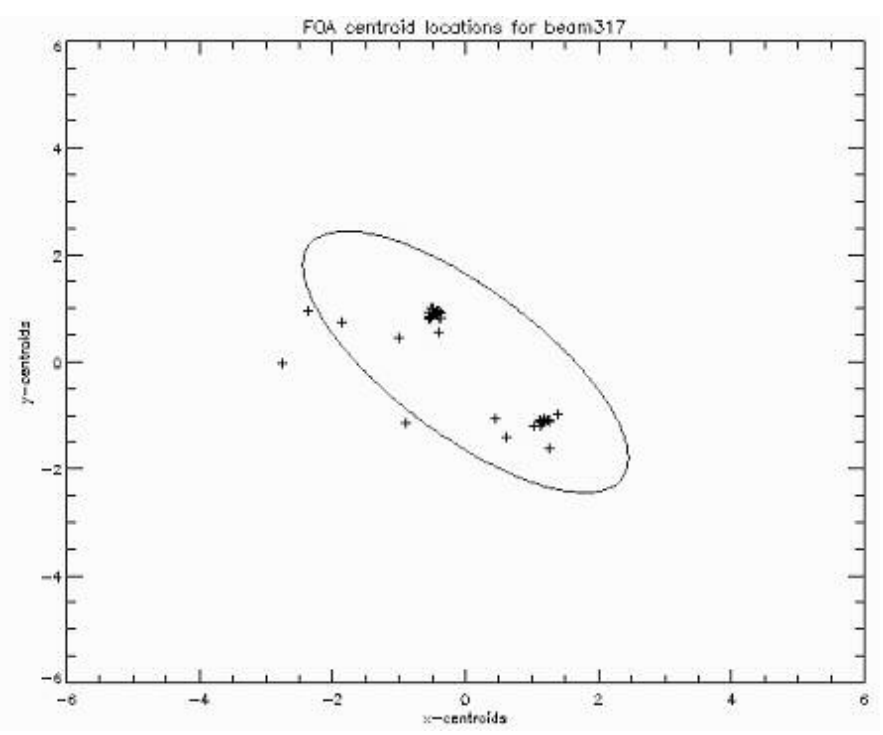

Figure 5. Two-sigma confidence ellipse for beam 317

\section{ANALYSIS OF ROBUSTNESS}

The uncertainty of the algorithm is evaluated by Monte Carlo simulation. In the simulation a real image was considered and noise of various rms values was added to different illumination cases. The illumination of the image was limited to three values of 50, 100, and 200 maximum amplitude. The noise rms was chosen as 10, 20, and 50 counts. One hundred images were created by adding Gaussian noise for each pair of amplitude and noise levels, resulting in a total of 900 images. For each 100-image set the 100 different positions are estimated, which theoretically should be at the same location with minimum variance. The standard deviation of these position estimates is multiplied by 3 to give a measure of uncertainty. As can be seen from Figure 6 (amplitude 200 and noise 20) the uncertainty is slightly over 0.2 pixels. The original algorithm gives a higher uncertainty of more than 0.5 pixels as shown in Figure 7. 


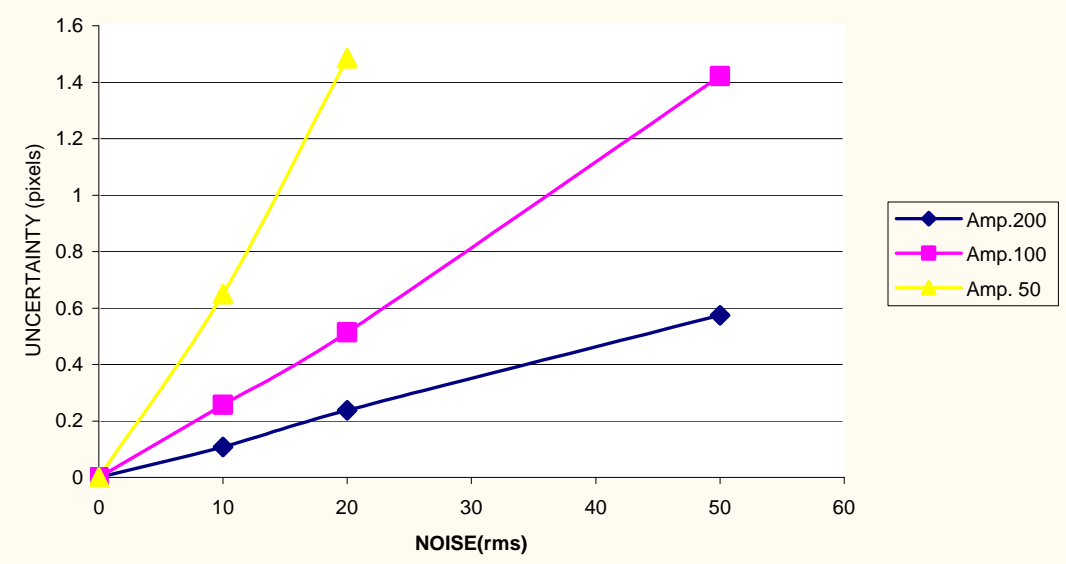

Figure 6. Output of the advanced FOA match filter algorithm at three-sigma standard deviations

The original FOA Corner Cube algorithm was based on a template match with the template extracted from a synthetic image. In the original algorithm two templates are needed, one for the upper spot and one for the lower. The main problem with the original algorithm is that images obtained from the real set up undergo a certain degree of transformation due to defocus and other phase aberrations that cause the artificial template to deviate from the real image.

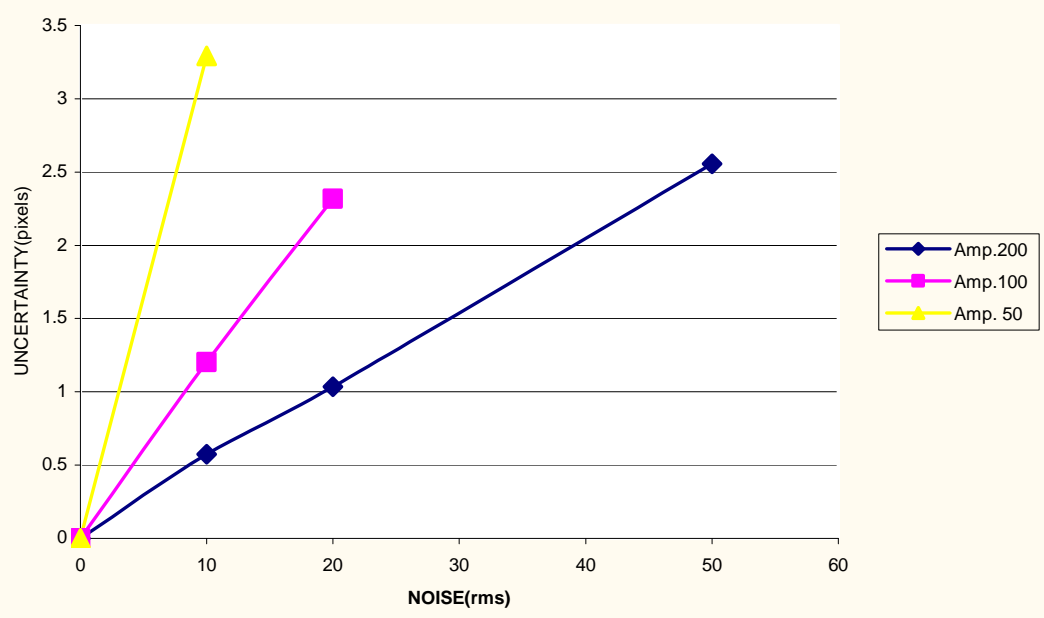

Figure 7. Uncertainty curves for the original FOA algorithm

The noise and uncertainty graph in Figure 7 accurately depicts the dilemmas of the original FOA algorithm versus the proposed algorithm. The algorithm yielded a high uncertainty in the presence of high amplitude signal counts with a low 
noise count. For example, at amplitude 200 and noise count 20 the uncertainty is approximately 1.1 pixels. The uncertainty at amplitude 200 and noise count 20 exceeds the three-sigma guidelines of 0.5 pixels. By examining graph plots for varying amplitude and noise count images, it is clear that the original FOA algorithm is neither very robust nor accurate. In order to improve precision, the correlation operation was performed with a 5 times expanded template. This expansion introduced interpolation error. Additionally, in the original FOA algorithm, the Sobel mask was used to find the gradient of the image before the template match. By differentiating the image, noise was intensified as demonstrated in the correlation plane output shown in Figure 8. The intensified noise lowers the accuracy and robustness of the original FOA algorithm. Moreover, by operating in the spatial domain the algorithm had a very slow run time of 33 seconds. On the other hand the new algorithm with an optimal template acheived a smoother correlation plane.

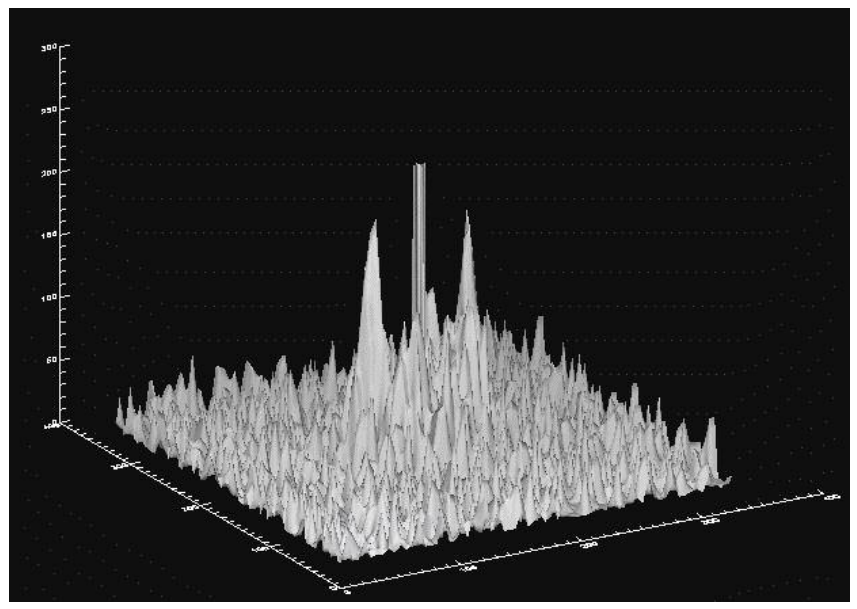

Figure 8. Original FOA algorithm performance

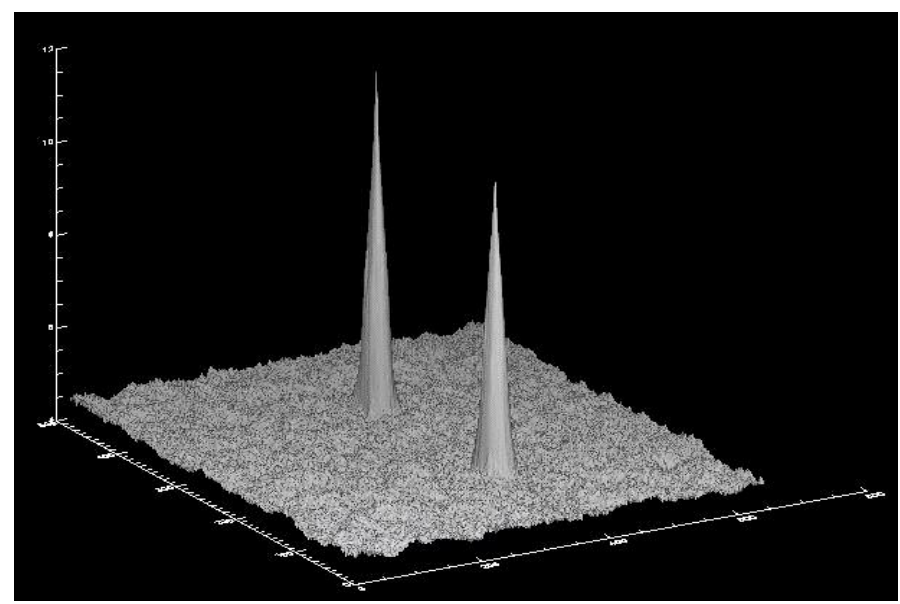

Figure 9. Advanced FOA algorithm performance

\section{CONCLUSIONS}

We have developed an algorithm for FOA corner cube position detection and tested it with 900 simulated noisy images. For these tests the template was chosen from a series of images taken from NIF over a year. The results show that for the best-case scenario (amplitude 200, noise count 10), the three-sigma noise is about 0.1 pixels. One worst case (amplitude 50, noise 20) shows the three-sigma value is 3.7 pixels. The Advanced FOA Match Filter algorithm is clearly more robust. The accuracy and robustness of the algorithm was achieved by fabricating an optimal template by averaging over a one-year cycle and by rejecting outliers that may bias the positioning and accuracy of the template using a $95 \%$ confidence interval.

\section{ACKNOWLEDGEMENT}

This work was performed under the auspices of the U.S. Department of Energy by the University of California, Lawrence Livermore National Laboratory under contract No. W-7405-Eng-48.

\section{REFERENCES}

1. M. Rahman, A. A. S. Awwal, and K. Gudmundsson, "Composite Filters for Search Time Reduction for 3D Model Based Object Recognition," Photonic Devices and Algorithms for Computing V, K.M. Iftekharuddin and A. A. S. Awwal Editors, Proc. of SPIE, Vol. 4201, 2003.

2. A. A. S. Awwal, M. A. Karim, and S. R. Jahan, "Improved Correlation Discrimination Using an Amplitudemodulated Phase-Only Filter," Applied Optics, Vol. 29, pp. 233-236, 1990. 